\title{
Diabetes does not impact the diagnostic performance of contrast-based fractional flow reserve: insights from the CONTRAST study
}

\author{
Giuseppe Gargiulo ${ }^{1}$, Eugenio Stabile , Marco Ferrone ${ }^{1}$, Emanuele Barbato ${ }^{1,2}$, Frederik M. Zimmermann³, \\ Julien Adjedj ${ }^{2}$, Barry Hennigan ${ }^{4,5}$, Mitsuaki Matsumura ${ }^{6}$, Nils P. Johnson7, William F. Fearon ${ }^{8,9}$, Allen Jeremias ${ }^{6,10}$, \\ Bruno Trimarco ${ }^{1}$, Giovanni Esposito ${ }^{1,11^{*}}$ and On behalf of the CONTRST Study Investigators
}

\begin{abstract}
Background: Adenosine-free coronary pressure wire metrics have been proposed to test the functional significance of coronary artery lesions, but it is unexplored whether their diagnostic performance might be altered in patients with diabetes.

Methods: We performed a post-hoc analysis of the CONTRAST study, which prospectively enrolled an international cohort of patients undergoing routine fractional flow reserve (FFR) assessment for standard indications. Paired, repeated measurements of all physiology metrics (Pd/Pa, iFR, contrast-based FFR, and FFR) were made. A central core laboratory analyzed blinded pressure tracings in a standardized fashion.

Results: Of 763 subjects enrolled at 12 international centers, 219 (29\%) had diabetes. The two groups were wellbalanced for age, clinical presentation (stable or unstable), coronary vessel studied, volume and type of intracoronary contrast, and volume of intracoronary adenosine. A binary threshold of cFFR $\leq 0.83$ produced an accuracy superior to both $\mathrm{Pd} / \mathrm{Pa}$ and iFR when compared with FFR $\leq 0.80$ in the absence of significant interaction with diabetes status; indeed, accuracy in subgroups of patients with or without diabetes was similar for CFFR (86.7 vs $85.4 \%$ respectively; $p=0.76)$, iFR (84.2 vs $80.0 \%, p=0.29)$ and $\mathrm{Pd} / \mathrm{Pa}(81.3$ vs $78.9 \%, p=0.55)$. There was no significant heterogeneity between patients with or without diabetes in terms of sensitivity and specificity of all metrics. The area under the receiver operating characteristic (ROC) curve was largest for cFFR compared with $\mathrm{Pd} / \mathrm{Pa}$ and iFR which were equivalent (CFFR 0.961 and 0.928; Pd/Pa 0.916 and 0.870; iFR 0.911 and 0.861 in diabetic and non-diabetic patients respectively).
\end{abstract}

Conclusions: CFFR provides superior diagnostic performance compared with Pd/Pa or iFR for predicting FFR irrespective of diabetes (clinicaltrials.gov identifier NCT02184117).

Keywords: Diabetes, Coronary lesion, Fractional flow reserve, Hyperemia, Contrast medium, Adenosine, Resting metrics, Instantaneous wave-free ratio

\section{Background}

Due to its multi-test validation and cost-effective improvement in clinical outcomes demonstrated by multiple randomized trials as well as observational data,

\footnotetext{
${ }^{*}$ Correspondence: espogiov@unina.it

${ }^{11}$ Division of Cardiology-Department of Advanced Biomedical Sciences,

Federico II University of Naples, Via Pansini 5, 80131 Naples, Italy

Full list of author information is available at the end of the article
}

physiological assessment of coronary stenoses by fractional flow reserve (FFR) has emerged as the gold standard for making revascularization decisions on stable lesions [1-5]. Importantly, FFR requires hyperemia, but that does add a small cost and risk [1, 6, 7]. Therefore, the CONTRAST (Can cONTrast Injection Better Approximate FFR compAred to Pure reSTing Physiology?) study recently investigated whether contrast medium could provide an easy alternative and inexpensive tool for 
assessing FFR (namely contrast FFR, cFFR). It demonstrated that CFFR was superior to resting measurements (rest distal pressure $[\mathrm{Pd}] /$ aortic pressure $[\mathrm{Pa}]$, and the instantaneous wave-free ratio [iFR]) in terms of diagnostic performance to predict FFR [8].

Diabetes mellitus increases cardiovascular risk, which has been attributed mainly to its detrimental effects on vascular function [9-13]. Before contributing to the development of structural vascular changes or significant coronary artery disease (CAD), diabetes impairs endothelial function leading to microvascular dysfunction [1218]. Even in diabetic patients without additional cardiac risk factors, endothelial dysfunction has been demonstrated and explained by associated autonomic dysfunction, chronic hyperglycemia, and insulin resistance.

Diabetes does not seem to significantly impact FFR accuracy or its interpretation [4, 19-22], although it can produce coexisting epicardial lesions (quantified by FFR) and microvascular dysfunction (often quantified by measures of hyperemic resistance). Because of potential alterations in microvascular reactivity, cFFR might perform differently in diabetic patients. Thus the aim of this study was to explore whether diabetes might impact the diagnostic ability of cFFR compared with $\mathrm{Pd} / \mathrm{Pa}$ or $\mathrm{iFR}$ versus adenosine-derived $\mathrm{FFR} \leq 0.80$.

\section{Methods}

\section{Study population}

We explored the impact of diabetes in a post-hoc analysis of the CONTRAST study (clinicaltrials.gov identifier NCT02184117). The design and results of this study have been previously published [8]. Briefly summarized, CONTRAST was an investigator-initiated, prospective diagnostic accuracy study that enrolled a multicenter, international cohort of patients undergoing routine FFR assessment for standard indications. Patients were recruited from 12 centers between June 2014 and April 2015. Subsequent care proceeded as per routine from the clinical FFR measurement without further studyrelated follow-up. Each subject gave informed consent as approved by the local institutional review board of that participating center. Subjects were excluded in case of prior coronary bypass surgery, known severe cardiomyopathy (LV ejection fraction $<30 \%$ ) or LV hypertrophy (septal wall thickness $>13 \mathrm{~mm}$ ), contraindication to adenosine, or renal insufficiency such that an additional $12-20 \mathrm{ml}$ of contrast would, in the opinion of the operator, pose an unwarranted risk. In cases of multivessel disease, only the first lesion studied using FFR was included. Culprit lesions for an acute infarction were excluded, but non-culprit lesions were permitted. Standard demographic, clinical, and catheterization parameters were collected for each subject.

\section{Measurements and core lab analysis}

The physiology protocol and core lab analysis used for the study has been previously described [8]. Briefly, an initial period of at least $1 \mathrm{~min}$ provided a stable assessment of resting physiology without further contrast injection. Next, a manual or injector-based IC bolus of contrast medium was given as per local practice for diagnostic angiography. The volume and type of IC contrast medium were not mandated but varied among sites and even among subjects at a single site but with a strong recommendation for 6-10 $\mathrm{ml}$. A second IC bolus of contrast medium was injected using the same technique when conditions had returned to baseline (after approximately $1 \mathrm{~min}$ ). Next, after the return of baseline conditions, a manual IC bolus of adenosine was administered (dose at operators' discretion but with a strong recommendation for 100-200 $\mu \mathrm{g}$ ). A second, identical bolus of IC adenosine was given after $1 \mathrm{~min}$.

A subsequent period of at least 1 min provided a second assessment of resting physiology before starting an adenosine infusion at a standard rate of $140 \mu \mathrm{g} / \mathrm{kg} / \mathrm{min}$ via either a central or wide bore peripheral IV. The duration of the infusion was approximately $2 \mathrm{~min}$, but could be prolonged or abbreviated as necessary. After stopping IV adenosine and waiting approximately $2 \mathrm{~min}$ for conditions to return to baseline, a second, identical IV adenosine infusion was performed. At the end of the procedure, an optional but recommended drift check was performed by bringing the pressure sensor back to the guide catheter at the same location as equalization.

All pressure tracings were sent to the Cardiovascular Research Foundation physiology core lab for standardized and centralized review. The core lab carried out its post-hoc analysis without knowledge of the locally determined $\mathrm{Pd} / \mathrm{Pa}$ value, IC substance (contrast medium or adenosine), enrolling site, or subject/lesion characteristics.

\section{Statistical analysis and endpoints}

The primary endpoint was accuracy against FFR $\leq 0.80$ and compared using a McNemar test between metrics. Secondary endpoints included the area under the receiver operating characteristic (ROC) curve (compared using the DeLong method), sensitivity, and specificity. All the analyses were performed in diabetic and non-diabetic patients. Based on previous evidence [8], the binary thresholds were as follows: $\mathrm{FFR} \leq 0.80$; cFFR $\leq 0.83$; $\mathrm{Pd} /$ $\mathrm{Pa}<0.92$ and $\mathrm{iFR}<0.90$.

Continuous variables are presented as mean \pm standard deviation and were compared with independent samples Student $t$ test. Categorical variables are expressed as count and percentages and were compared with Chi square or Fisher exact tests as appropriate. A Cox 
proportional model was used for univariate and multivariate analysis to explore the role of diabetes in predicting the cFFR disagreement with FFR. Applicable tests were two-tailed, and $\mathrm{p}<0.05$ was considered statistically significant. Analyses were performed in SPSS, version 23.0 (SPSS Inc., Chicago, IL, USA) and MedCalc version 12.5 (MedCalc Software, Ostend, Belgium).

\section{Results}

Of 763 subjects, 219 (29\%) were diabetic. Compared with non-diabetic patients, those with diabetes were more frequently women, had a higher body mass index, and more frequently had hypertension or dyslipidemia (Table 1). The 2 groups were well-balanced for clinical presentation (stable or unstable), coronary vessel studied, volume and type of intracoronary contrast, and volume of intracoronary adenosine (Table 1 ).

As reported previously, a binary threshold of cFFR $\leq 0.83$ produced an accuracy of $85.8 \%$, superior to both $\mathrm{Pd} / \mathrm{Pa} 78.5 \%$ and iFR $79.9 \%$ (McNemar $\mathrm{p}<0.001$ versus both resting metrics), when compared with FFR $\leq 0.8$ [8]. In this substudy, there was no significant interaction between diabetes and accuracy; indeed, accuracy in subgroups of patients with or without diabetes was similar for cFFR ( 86.7 vs $85.4 \%$ respectively; $\mathrm{p}=0.76)$, iFR ( 84.2 vs $80.0 \%$ respectively; $\mathrm{p}=0.29)$ and $\mathrm{Pd} / \mathrm{Pa}(81.3$ vs $78.9 \%$ respectively; $\mathrm{p}=0.55$ ) (Fig. 1 ). Overall, sensitivity was similar among the 3 metrics when comparing non-diabetics and diabetics, respectively (cFFR 78.6 vs $74.7 \%, \mathrm{p}=0.61$; iFR 88.8 vs $77.2 \%, \mathrm{p}=0.05$; $\mathrm{Pd} / \mathrm{Pa} 78.8$ vs $74.6 \%, \mathrm{p}=0.57$ ), but cFFR improved specificity compared with iFR or $\mathrm{Pd} / \mathrm{Pa}$ and there was no significant interaction with diabetes status (cFFR 95.6 vs $95.3 \%, \mathrm{p}=0.85$; iFR 78.9 vs $78.6 \%, \mathrm{p}=0.89$; $\mathrm{Pd} / \mathrm{Pa} 84.0$ vs $82.9 \%, \mathrm{p}=0.96$ ) (Fig. 1).

The area under the ROC curve was largest for cFFR compared with $\mathrm{Pd} / \mathrm{Pa}$ and iFR which were equivalent in both diabetic (0.961 cFFR, $0.916 \mathrm{Pd} / \mathrm{Pa}, 0.911 \mathrm{iFR}$; DeLong $\mathrm{p}=0.003$ for cFFR vs iFR, $\mathrm{p}<0.0001$ for $\mathrm{cFFR}$ vs $\mathrm{Pd} / \mathrm{Pa}$ and $\mathrm{p}=0.66$ for iFR vs $\mathrm{Pd} / \mathrm{Pa}$; Fig. 2) and nondiabetic patients $(0.928 \mathrm{cFFR}, 0.870 \mathrm{Pd} / \mathrm{Pa}, 0.861 \mathrm{iFR}$; DeLong $\mathrm{p}<0.0001$ for cFFR vs iFR, $\mathrm{p}<0.0001$ for $\mathrm{cFFR}$ vs $\mathrm{Pd} / \mathrm{Pa}$ and $\mathrm{p}=0.13$ for iFR vs $\mathrm{Pd} / \mathrm{Pa}$; Fig. 2). There were no significant differences when ROC curves in diabetic and non-diabetic patients were compared (cFFR 0.961 vs 0.928 , DeLong $\mathrm{p}=0.08 ; \mathrm{Pd} / \mathrm{Pa} 0.916$ vs 0.870 , DeLong $\mathrm{p}=0.09$; iFR 0.911 vs 0.861 , DeLong $\mathrm{p}=0.08$ ).

As a physiologic explanation for the superior diagnostic performance of cFFR compared to resting physiology (either $\mathrm{Pd} / \mathrm{Pa}$ or iFR), Fig. 3 shows that the modest hyperemia induced by cFFR determines a more linear and less scattered relationship versus FFR compared to that of resting physiology, irrespective of diabetes status.
Indeed, compared with $\mathrm{Pd} / \mathrm{Pa}$ and iFR, the correlation of cFFR with FFR was superior in both diabetic $(r=0.92$ and ICC $=0.85$ for cFFR; $r=0.84$ and $\mathrm{ICC}=0.64$ for iFR; $r=0.87$ and $\mathrm{ICC}=0.37$ for $\mathrm{Pd} / \mathrm{Pa}$ ) or non-diabetic patients $(\mathrm{r}=0.93$ and $\mathrm{ICC}=0.84$ for $\mathrm{cFFR} ; \mathrm{r}=0.80$ and $\mathrm{ICC}=0.56$ for $\mathrm{iFR} ; \mathrm{r}=0.85$ and $\mathrm{ICC}=0.38$ for $\mathrm{Pd} / \mathrm{Pa}$; Fig. 3).

Diabetes did not predict agreement/disagreement between cFFR and FFR $(\mathrm{p}=0.66)$ in a univariate analysis. Also when diabetes was forced into a multivariable model, it did not significantly influence the accuracy of cFFR (adjusted $\mathrm{p}=0.71$ ) (Table 2).

\section{Discussion}

This sub-analysis of the CONTRAST study shows that there is no significant interaction between diabetes and the diagnostic performance of cFFR, therefore, the findings of the main analysis can be extended to patients with diabetes. Contrast FFR provides superior diagnostic performance compared with resting metrics (iFR and $\mathrm{Pd} / \mathrm{Pa}$ ) irrespective of diabetic status.

The CONTRAST study is the largest study to investigate the diagnostic performance of cFFR and the present sub-analysis is the first to explore the impact of diabetes on cFFR performance compared with resting methods [23-25].

Diabetes mellitus is a well-known risk factor for cardiovascular disease [9-11]. The presence of diabetes accelerates the natural course of atherosclerosis, involving both proximal epicardial and distal small vessel coronary arteries with greater plaque ulceration and thrombosis and microvascular disease. Patients with diabetes have a variety of structural and functional microvascular coronary abnormalities [12-18] that may translate into a reduction of hyperemic blood flow due to an impairment of microcirculatory vasodilation.

Some studies investigated the accuracy of FFR in diabetic patients. Yanagisawa et al. compared FFR performance in 96 and 149 patients with or without diabetes respectively and observed that there was no significant difference, although within diabetic patients, those with a poor glycemic control (glycated hemoglobin $>7 \%$ ) had a lower FFR specificity [19]. Domínguez-Franco et al. found that deferring PCI in diabetic patients (29\% of all patients) with a moderately severe coronary artery stenosis based on the FFR value was as safe as in patients without diabetes [20]. Sahinarslan et al. analyzed 122 patients (29.5\% with diabetes) with intermediate coronary lesions and observed that there was no difference between the FFR values in patients with or without diabetes who had been paired according to reference vessel diameter and degree of luminal narrowing of coronary lesions [21]. Reith et al. evaluated FFR in 266 intermediate grade 
Table 1 Baseline characteristics in patients with or without diabetes

\begin{tabular}{|c|c|c|c|}
\hline & No diabetes $(\mathrm{N}=544)$ & Diabetes $(N=219)$ & $p$ value \\
\hline Age (years) & $65.4 \pm 9.7$ & $66.4 \pm 9.7$ & 0.24 \\
\hline Male & $74.8 \%(407)$ & $63.9 \%(140)$ & 0.003 \\
\hline BMI $\left(\mathrm{kg} / \mathrm{m}^{2}\right)$ & $26.8 \pm 4.5$ & $28.6 \pm 5.0$ & $<0.0001$ \\
\hline Smoking (current or past) & $47.8 \%(260)$ & $47.0 \%(103)$ & 0.85 \\
\hline Hypertension & $67.5 \%(367)$ & $81.3 \%(178)$ & $<0.0001$ \\
\hline Dyslipidemia & $61.4 \%(334)$ & $79.5 \%(174)$ & $<0.0001$ \\
\hline Family history of CAD & $26.1 \%(142)$ & $22.4 \%(49)$ & 0.28 \\
\hline Renal dysfunction (eGFR < $60 \mathrm{ml} / \mathrm{min}$ ) & $8.6 \%(47)$ & $12.3 \%(27)$ & 0.12 \\
\hline Prior Ml & $24.1 \%(131)$ & $30.6 \%(67)$ & 0.06 \\
\hline Prior PCl & $14.0 \%(76)$ & $17.4 \%(38)$ & 0.24 \\
\hline Peripheral vascular disease & $3.7 \%(20)$ & $6.4 \%(14)$ & 0.10 \\
\hline Clinical presentation & & & 0.37 \\
\hline Stable & $79.2 \%(431)$ & $76.3 \%(167)$ & \\
\hline ACS & $20.8 \%(113)$ & $23.7 \%(52)$ & \\
\hline Unstable Angina & $9.2 \%(50)$ & $15.5 \%(34)$ & \\
\hline NSTEMI & $10.7 \%(58)$ & $6.8 \%(15)$ & \\
\hline STEMI & $0.9 \%(5)$ & $1.4 \%(3)$ & \\
\hline Coronary vessel & & & 0.86 \\
\hline Left main & $3.1 \%(17)$ & $3.7 \%(8)$ & \\
\hline LAD & $60.7 \%(330)$ & $59.4 \%(130)$ & \\
\hline LCx & $17.5 \%(95)$ & $19.6 \%(43)$ & \\
\hline $\mathrm{RCA}$ & $18.8 \%(102)$ & $17.4 \%(38)$ & \\
\hline Contrast medium & & & 0.03 \\
\hline lobitridol & $5.3 \%(29)$ & $5.0 \%(11)$ & \\
\hline lodixanol & $24.3 \%(132)$ & $26.0 \%(57)$ & \\
\hline lohexol & $15.8 \%(86)$ & $9.1 \%(20)$ & \\
\hline lomeprol & $31.1 \%(169)$ & $26.5 \%(58)$ & \\
\hline lopamidol & $1.3 \%(7)$ & $0.5 \%(1)$ & \\
\hline lopromide & $7.9 \%(43)$ & $11.9 \%(26)$ & \\
\hline loversol & $8.5 \%(46)$ & $10.0 \%(22)$ & \\
\hline loxaglate & $5.9 \%(32)$ & $11.0 \%(24)$ & \\
\hline Volume of IC contrast (ml) & $7.9 \pm 1.5$ & $7.7 \pm 1.6$ & 0.03 \\
\hline 5 & $2.0 \%(11)$ & $2.7 \%(6)$ & \\
\hline $6-7$ & $39.7 \%(216)$ & $49.3 \%(108)$ & \\
\hline $8-9$ & $30.1 \%(164)$ & $21.0 \%(46)$ & \\
\hline 10 & $27.9 \%(152)$ & $26.9 \%(59)$ & \\
\hline 12 & $0.2 \%(1)$ & $0 \%(0)$ & \\
\hline Dose of IC adenosine* $(\mu \mathrm{g})$ & $166.3 \pm 46.1$ & $171.2 \pm 47.1$ & 0.27 \\
\hline$<80$ & $2.3 \%(9)$ & $1.3 \%(2)$ & \\
\hline $80-90$ & $7.1 \%(28)$ & $7.1 \%(11)$ & \\
\hline $100-150$ & $28.4 \%(112)$ & $29.0 \%(45)$ & \\
\hline $160-200$ & $48.5 \%(191)$ & $45.2 \%(70)$ & \\
\hline$>200$ & $13.7 \%(54)$ & $17.4 \%(27)$ & \\
\hline
\end{tabular}

$A C S$ acute coronary syndrome, $B M I$ body mass index, CAD coronary artery disease, GFR glomerular filtration rate, IC intracoronary, $L A D$ left anterior descending coronary artery, $L C X$ left circumflex coronary artery, $M I$ myocardial infarction, NSTEMI non ST-segment elevation $\mathrm{MI}, P C I$ percutaneous coronary intervention, $R C A$ right coronary artery, STEMI ST-segment elevation MI

* Only 549 of 763 patients received IC adenosine, while all other rows are based on 763 total 

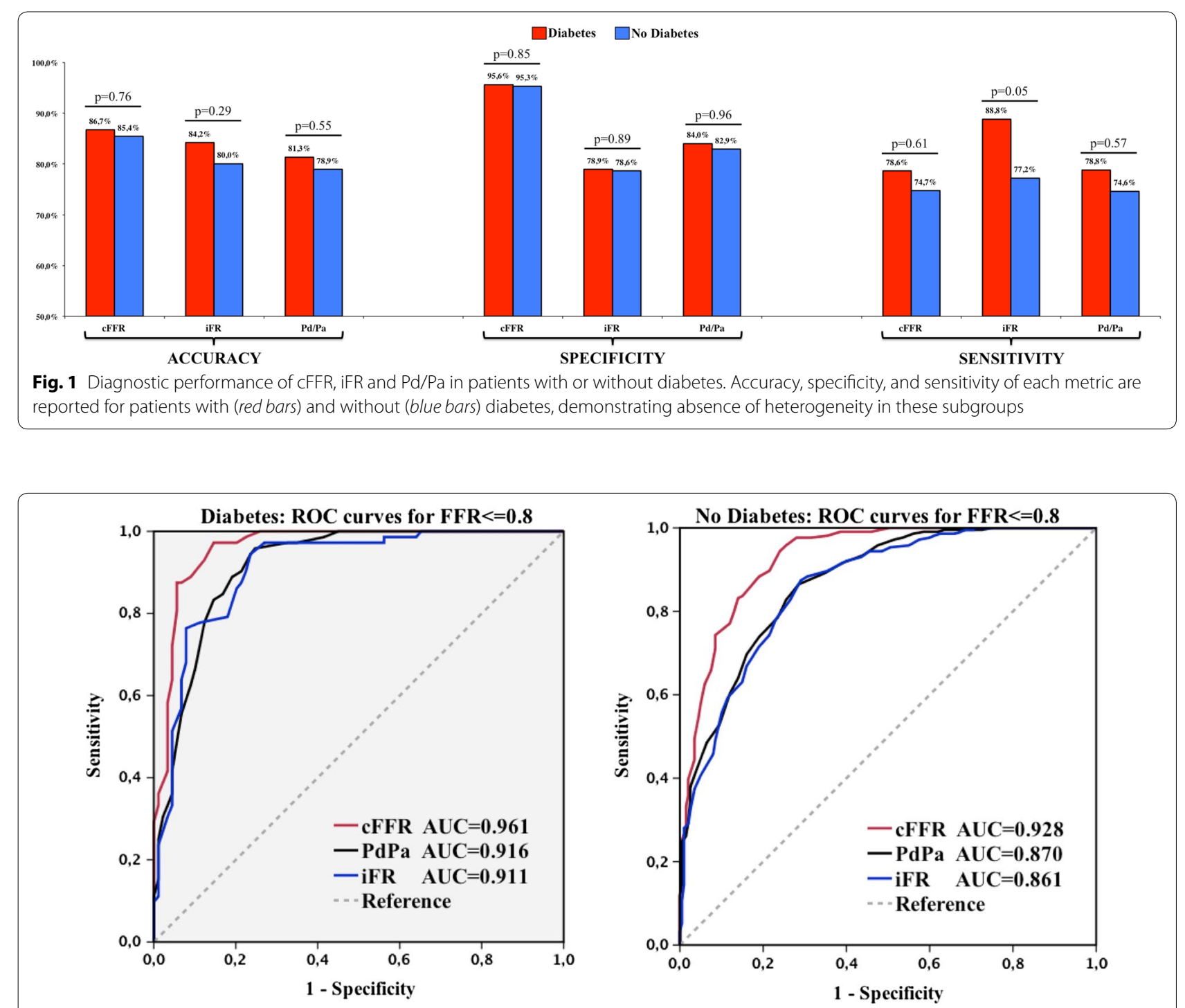

Fig. 2 Diagnostic performance expressed by the area under the ROC curve for each metric in patients with or without diabetes. In patients with or without diabetes, contrast FFR (red line) has the largest area under the ROC curve, while iFR (blue line) and Pd/Pa (black line) have equivalent areas

lesions of 224 patients (113 non-diabetics and 111 diabetics) with stable CAD, also exploring the role of adequate glycemic control (defined as glycated hemoglobin $<7 \%$ ). They found that FFR accuracy was not affected by diabetic status and its glycemic control [22]. In the FAME-2 trial, the FFR guided PCI was superior to medical therapy for the primary end point (composite of death from any cause, nonfatal myocardial infarction, or urgent revascularization within 2 years) in patients with or without diabetes (28 and $72 \%$ respectively) with an absence of significant interaction between the two sub-groups interaction $\mathrm{p}=0.50)[4]$.

Interestingly, some data have questioned the accuracy of FFR is some cases. Although FFR and coronary flow velocity reserve (CFVR) have an equivalent diagnostic accuracy for inducible myocardial ischemia, it has been shown that they provide discordant results in $30-40 \%$ of cases, which was suggested to have a significant impact on clinical outcomes, particularly when FFR is normal and CFVR abnormal that generally indicates predominant microvascular involvement in coronary artery disease $[26,27]$. Therefore it cannot be excluded that the accuracy of FFR, and thus also of cFFR, may be affected in case of microvascular disease as occurs in patients with diabetes. Indeed, a recent study has showed that in patients with diabetes, particularly those with previous MI, FFR-based deferred revascularization was associated with poor medium-term outcomes suggesting that 


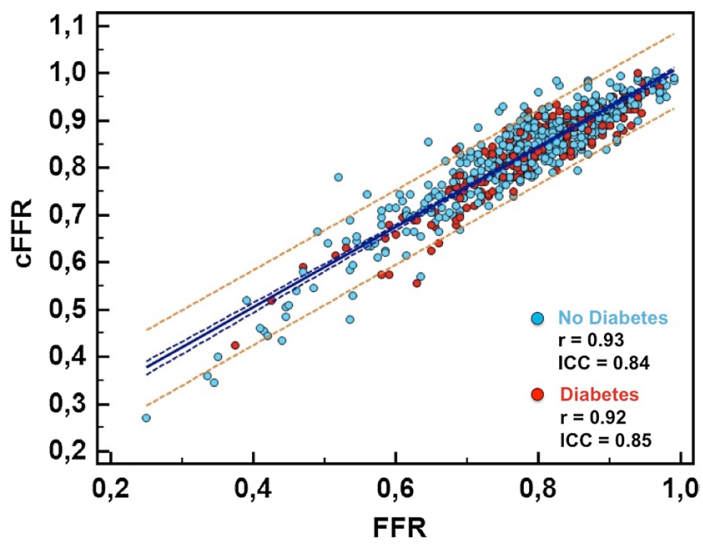

Table 2 Predictors of cFFR disagreement with FFR

\begin{tabular}{lll}
\hline & $\begin{array}{l}\text { Univariate } \\
\text { p value }\end{array}$ & $\begin{array}{l}\text { Multivariate } \\
\text { p value }\end{array}$ \\
\hline Age (years) & 0.06 & 0.34 \\
Male & 0.54 & \\
BMI (kg/m²) & 0.11 & 0.59 \\
Diabetes & 0.66 & 0.71 \\
Smoking (current or past) & 0.59 & \\
Hypertension & 0.50 & \\
Dyslipidemia & 0.36 & \\
Family history of CAD & 0.87 & \\
Renal dysfunction (eGFR $<60$ ml/min) & 0.09 & 0.30 \\
Prior MI & 0.58 & \\
Prior PCl & 0.89 & \\
Peripheral vascular disease & 0.42 & \\
Clinical presentation (stable or ACS) & 0.89 & \\
Coronary vessel & 0.045 & 0.89 \\
Syntax segment & 0.004 & 0.26 \\
Contrast medium & 0.004 & 0.44 \\
Volume of IC contrast & 0.005 & 0.37 \\
Dose of IC adenosine* & 0.038 & 0.76 \\
\hline ACS & & \\
\hline
\end{tabular}

$A C S$ acute coronary syndrome, $B M I$ body mass index, $C A D$ coronary artery

disease, GFR glomerular filtration rate, IC intracoronary, $M I$ myocardial infarction, $\mathrm{PCl}$ percutaneous coronary intervention

* Only 549 of 763 patients received IC adenosine, while all other rows are based on 763 total

FFR-guided deferred revascularization in patients with and without diabetes, Kennedy et al. also showed that those with diabetes were associated with a significantly higher rate of target lesion failure [29]. Accordingly, a recent study by Liu et al. showed that a higher FFR was associated with lower rates of death, myocardial infarction and revascularization among non-diabetic patients with deferred PCI, but in diabetic patients with deferred revascularization, FFR was not able to differentiate the risk of cardiovascular events [30].

Whether intravascular imaging, may impact on this higher failure rate remains unexplored, but the COMBINE study, the first prospective multicenter combining FFR and optical coherence tomography, will contribute to clarify this issue investigating the hypothesis that adding the study of plaque morphology to FFR in intermediate lesions may better predict events in patients with diabetes [31].

Although few data exist on the clinical value of FFR in diabetic patients, the impact of diabetes and any potentially associated microvascular dysfunction was previously unknown for cFFR performance. Indeed, whether the submaximal vasodilatation induced by contrast medium compared with adenosine might affect the accu-

racy of cFFR measurement in patients with diabetes had useful to guide our treatment strategy in these patients with high-risk, fast-progressing disease [28]. Comparing 
never been explored before. Our novel results found no significant heterogeneity between subgroups stratified by diabetes, and that the overall results of the CONTRAST study can be extended to all patients irrespective of diabetic status. Therefore, cFFR has been proven accurate and superior to resting metrics in patients with diabetes as well as in those without diabetes.

\section{Limitations}

This is an unplanned post-hoc analysis of the CONTRAST study, which was not designed or powered to investigate the diabetic subgroup specifically. Therefore, our exploratory analysis should be considered hypothesis-generating and needs to be confirmed in further trials. Additionally, our sub-analysis shares the limitations of the main study [8], namely the short-acting nature of contrast-induced hyperemia and lack of clinical data regarding contrast-induced nephropathy. Finally, being cFFR measured during submaximal hyperemia, it shares the same FFR limitations related to hyperemia in the setting of microvascular dysfunction.

\section{Clinical relevance and conclusions}

When performing physiological lesion assessment, some operators prefer to avoid adenosine due to rare side effects and minimal but nonzero added time and expense. Moreover, adenosine is still expensive or unavailable in some areas of the world, and sporadic patients have contraindications.

Contrast FFR provides superior diagnostic performance than $\mathrm{Pd} / \mathrm{Pa}$ or iFR for predicting FFR irrespective of diabetes. Although FFR remains the reference standard for diagnostic certainty, for clinical scenarios or healthcare systems in which adenosine is contraindicated or prohibitively expensive, cFFR may represent a simple alternative technique to adenosine-FFR for invasive coronary physiological assessment in diabetic as well as nondiabetic patients.
${ }^{4}$ West of Scotland Heart and Lung Centre, Golden Jubilee National Hospital, Clydebank, Scotland, UK. ${ }^{5}$ British Heart Foundation Glasgow Cardiovascular Research Centre, Institute of Cardiovascular and Medical Sciences, University of Glasgow, Glasgow, Scotland, UK. ${ }^{6}$ Cardiovascular Research Foundation (CRF), New York, NY, USA. ${ }^{7}$ Division of Cardiology, Department of Medicine, Weatherhead PET Center, McGovern Medical School at UTHealth and Memorial Hermann Hospital, Houston, TX, USA. ${ }^{8}$ Stanford University Medical Center, Stanford, USA. ${ }^{9}$ The Palo Alto VA Health Care Systems, Palo Alto, CA, USA.

${ }^{10}$ Division of Cardiovascular Medicine, Stony Brook University Medical Center, Stony Brook, NY, USA. ${ }^{11}$ Division of Cardiology-Department of Advanced Biomedical Sciences, Federico II University of Naples, Via Pansini 5, 80131 Naples, Italy.

\section{Acknowledgements}

The authors thank all the centers and personal participating at the data collection.

\section{Competing interests}

Dr. Gargiulo is supported by a research grant from Cardiopath PhD program. Dr. Johnson has received internal funding from the Weatherhead PET Center for Preventing and Reversing Atherosclerosis; and significant institutional research support from St. Jude Medical (for this study [NCT02184117]) and Volcano/Philips Corporation (for NCT02328820), makers of intracoronary pressure and flow sensors. Dr. Jeremias has served as a consultant and member of the Speakers' Bureau for Volcano/Philips Corporation. Dr. Barbato has received institutional consultancy fees and research support from St. Jude Medical. Dr. Fearon has received institutional research support from St. Jude Medical and Medtronic; has received honoraria from Medtronic; and has served as a consultant to HeartFlow. All other authors have reported that they have no relationships relevant to the contents of this paper to disclose.

\section{Availability of data and material}

The datasets during and/or analyzed during the current study are available from the corresponding author on reasonable request.

\section{Consent for publication}

All authors have read and approved the submission of the manuscript; the manuscript has not been published and is not being considered for publication elsewhere, in whole or in part, in any language, except as an abstract.

\section{Ethics approval and consent to participate}

The study was approved by the local institutional review board of all participating centers. All participants gave their written informed consent before data collection.

\section{Funding}

The present sub-analysis did not receive financial support.

Received: 30 October 2016 Accepted: 30 December 2016

Published online: 13 January 2017

\begin{abstract}
Abbreviations
CAD: coronary artery disease; CFFR: contrast-based fractional flow reserve; ECG: electrocardiogram; FFR: fractional flow reserve; IC: intracoronary; iFR: instantaneous wave-free ratio; IQR: interquartile range; IV: intravenous(ly); Pd/ Pa: resting ratio of distal coronary pressure to aortic pressure; ROC: receiver operating characteristic curve.
\end{abstract}

\section{Authors' contributions}

GG, GE, BT NPJ, and WFF conceived and designed the study. GG performed all analyses, interpreted the results, and drafted the manuscript. GE, BT NPJ, and WFF interpreted the results, and revised critically the manuscript. All authors participated in data collection, revised critically the manuscript. All authors read and approved the final manuscript.

\section{Author details}

${ }^{1}$ Department of Advanced Biomedical Sciences, University of Naples Frederico II, Naples, Italy. ${ }^{2}$ Cardiovascular Center, OLV Clinic, Aalst, Belgium.

${ }^{3}$ Department of Cardiology, Catharina Hospital, Eindhoven, The Netherlands.

\section{References}

1. Pijls NH, De Bruyne B, Peels K, Van Der Voort PH, Bonnier HJ, Bartunek JKJJ, Koolen JJ. Measurement of fractional flow reserve to assess the functional severity of coronary-artery stenoses. N Engl J Med. 1996;334:1703-8.

2. Zimmermann FM, Ferrara A, Johnson NP, van Nunen LX, Escaned J, Albertsson P, Erbel R, Legrand V, Gwon HC, Remkes WS, Stella PR, van Schaardenburgh P, Bech GJ, De Bruyne B, Pijls NH. Deferral vs performance of percutaneous coronary intervention of functionally non-significant coronary stenosis: 15-year follow-up of the defer trial. Eur Heart J. 2015:36:3182-8.

3. van Nunen LX, Zimmermann FM, Tonino PA, Barbato E, Baumbach A, Engstrom T, Klauss V, MacCarthy PA, Manoharan G, Oldroyd KG, Ver Lee PN, Van't Veer M, Fearon WF, De Bruyne B, Pijls NH. Fractional flow reserve versus angiography for guidance of pci in patients with multivessel coronary artery disease (fame): 5-year follow-up of a randomised controlled trial. Lancet. 2015;386:1853-60. 
4. De Bruyne B, Fearon WF, Pijls NH, Barbato E, Tonino P, Piroth Z, Jagic N, Mobius-Winckler S, Rioufol G, Witt N, Kala P, MacCarthy P, Engstrom T, Oldroyd K, Mavromatis K, Manoharan G, Verlee P, Frobert O, Curzen N, Johnson JB, Limacher A, Nuesch E, Juni P. Fractional flow reserve-guided pci for stable coronary artery disease. N Engl J Med. 2014;371:1208-17.

5. Johnson NP, Gould KL, Di Carli MF, Taqueti VR. Invasive ffr and noninvasive cfr in the evaluation of ischemia: what is the future? J Am Coll Cardiol. 2016;67:2772-88.

6. Pijls NH, van Son JA, Kirkeeide RL, De Bruyne B, Gould KL. Experimental basis of determining maximum coronary, myocardial, and collateral blood flow by pressure measurements for assessing functional stenosis severity before and after percutaneous transluminal coronary angioplasty. Circulation. 1993;87:1354-67.

7. Johnson NP, Toth GG, Lai D, Zhu H, Acar G, Agostoni P, Appelman Y, Arslan F, Barbato E, Chen SL, Di Serafino L, Dominguez-Franco AJ, Dupouy P, Esen AM, Esen OB, Hamilos M, Iwasaki K, Jensen LO, Jimenez-Navarro MF, Katritsis DG, Kocaman SA, Koo BK, Lopez-Palop R, Lorin JD, Miller LH, Muller O, Nam CW, Oud N, Puymirat E, Rieber J, Rioufol G, Rodes-Cabau J, Sedlis SP, Takeishi Y, Tonino PA, Van Belle E, Verna E, Werner GS, Fearon WF, Pijls NH, De Bruyne B, Gould KL. Prognostic value of fractional flow reserve: linking physiologic severity to clinical outcomes. J Am Coll Cardiol. 2014:64:1641-54.

8. Johnson NP, Jeremias A, Zimmermann FM, Adjedj J, Witt N, Hennigan B, Koo BK, Maehara A, Matsumura M, Barbato E, Esposito G, Trimarco B, Rioufol G, Park SJ, Yang HM, Baptista SB, Chrysant GS, Leone AM, Berry C, De Bruyne B, Gould KL, Kirkeeide RL, Oldroyd KG, Pijls NH, Fearon WF. Continuum of vasodilator stress from rest to contrast medium to adenosine hyperemia for fractional flow reserve assessment. JACC CardiovasC Interv. 2016:9:757-67.

9. Beckman JA, Creager MA, Libby P. Diabetes and atherosclerosis: epidemiology, pathophysiology, and management. JAMA. 2002;287:2570-81.

10. Schramm TK, Gislason GH, Kober L, Rasmussen S, Rasmussen JN, Abildstrom SZ, Hansen ML, Folke F, Buch P, Madsen M, Vaag A, Torp-Pedersen C. Diabetes patients requiring glucose-lowering therapy and nondiabetics with a prior myocardial infarction carry the same cardiovascular risk: a population study of 3.3 million people. Circulation. 2008;117:1945-54.

11. Gargiulo G, Windecker S, da Costa BR, Feres F, Hong MK, Gilard M, Kim HS, Colombo A, Bhatt DL, Kim BK, Morice MC, Park KW, Chieffo A, Palmerini T, Stone GW, Valgimigli M. Short term versus long term dual antiplatelet therapy after implantation of drug eluting stent in patients with or without diabetes: systematic review and meta-analysis of individual participant data from randomised trials. BMJ. 2016:355:15483.

12. Marciano C, Galderisi M, Gargiulo P, Acampa W, D'Amore C, Esposito R, Capasso E, Savarese G, Casaretti L, Lo ludice F, Esposito G, Rengo G, Leosco D, Cuocolo A, Perrone-Filardi P. Effects of type 2 diabetes mellitus on coronary microvascular function and myocardial perfusion in patients without obstructive coronary artery disease. Eur J Nucl Med Mol Imaging. 2012;39:1199-206.

13. Picchi A, Capobianco S, Qiu T, Focardi M, Zou X, Cao JM, Zhang C. Coronary microvascular dysfunction in diabetes mellitus: a review. World J Cardiol. 2010;2:377-90.

14. Yokoyama I, Momomura S, Ohtake T, Yonekura K, Nishikawa J, Sasaki Y, Omata M. Reduced myocardial flow reserve in non-insulin-dependent diabetes mellitus. J Am Coll Cardiol. 1997;30:1472-7.

15. Pitkanen OP, Nuutila P, Raitakari OT, Ronnemaa T, Koskinen PJ, lida H, Lehtimaki TJ, Laine HK, Takala T, Viikari JS, Knuuti J. Coronary flow reserve is reduced in young men with iddm. Diabetes. 1998:47:248-54.

16. Nitenberg A, Valensi P, Sachs R, Dali M, Aptecar E, Attali JR. Impairment of coronary vascular reserve and ach-induced coronary vasodilation in diabetic patients with angiographically normal coronary arteries and normal left ventricular systolic function. Diabetes. 1993;42:1017-25.

17. Di Carli MF, Janisse J, Grunberger G, Ager J. Role of chronic hyperglycemia in the pathogenesis of coronary microvascular dysfunction in diabetes. J Am Coll Cardiol. 2003:41:1387-93.
18. Camici PG, Crea F. Coronary microvascular dysfunction. N Engl J Med. 2007;356:830-40

19. Yanagisawa H, Chikamori T, Tanaka N, Usui Y, Takazawa K, Yamashina A. Application of pressure-derived myocardial fractional flow reserve in assessing the functional severity of coronary artery stenosis in patients with diabetes mellitus. Circ J. 2004;68:993-8.

20. Dominguez-Franco AJ, Jimenez-Navarro MF, Munoz-Garcia AJ, AlonsoBriales JH, Hernandez-Garcia JM, de Teresa Galvan E. Long-term prognosis in diabetic patients in whom revascularization is deferred following fractional flow reserve assessment. Rev Esp Cardiol. 2008;61:352-9. doi:10.1016/S1885-5857(08)60144-9.

21. Sahinarslan A, Kocaman SA, Olgun H, KunakT, Kiziltunc E, Ozdemir M, Timurkaynak T. The reliability of fractional flow reserve measurement in patients with diabetes mellitus. Coron Artery Dis. 2009;20:317-21.

22. Reith S, Battermann S, Hellmich M, Marx N, Burgmaier M. Impact of type 2 diabetes mellitus and glucose control on fractional flow reserve measurements in intermediate grade coronary lesions. Clin Res Cardiol. 2014;103:191-201.

23. Kanaji Y, Murai T, Lee T, Matsuda J, Usui E, Araki M, Niida T, Ichijyo S, Hamaya R, Yonetsu T, Kimura S, Kakuta T. Efficacy of pressure parameters obtained during contrast medium-induced submaximal hyperemia in the functional assessment of intermediate coronary stenosis in comparison with instantaneous wave-free ratio. Int J Cardiol. 2016;208:128-36.

24. Leone AM, Scalone G, De Maria GL, Tagliaferro F, Gardi A, Clemente F, Basile E, Cialdella P, De Caterina AR, Porto I, Aurigemma C, Burzotta F, Niccoli G, Trani C, Rebuzzi AG, Crea F. Efficacy of contrast medium induced $\mathrm{pd} / \mathrm{pa}$ ratio in predicting functional significance of intermediate coronary artery stenosis assessed by fractional flow reserve: insights from the rinasci study. Eurolntervention. 2015;11:421-7.

25. De Bruyne B, Pijls NH, Barbato E, Bartunek J, Bech JW, Wijns W, Heyndrickx GR. Intracoronary and intravenous adenosine 5'-triphosphate, adenosine, papaverine, and contrast medium to assess fractional flow reserve in humans. Circulation. 2003;107:1877-83.

26. van de HoefTP, van Lavieren MA, Damman P, Delewi R, Piek MA, Chamuleau SA, Voskuil M, Henriques JP, Koch KT, de Winter RJ, Spaan JA, Siebes M, Tijssen JG, Meuwissen M, Piek JJ. Physiological basis and long-term clinical outcome of discordance between fractional flow reserve and coronary flow velocity reserve in coronary stenoses of intermediate severity. Circ Cardiovasc Interv. 2014;7:301-11.

27. Lee JM, Jung JH, Hwang D, Park J, Fan Y, Na SH, Doh JH, Nam CW, Shin ES, Koo BK. Coronary flow reserve and microcirculatory resistance in patients with intermediate coronary stenosis. J Am Coll Cardiol. 2016;67:1158-69.

28. Kennedy MW, Hermanides RS, Kaplan E, Hemradj V, Fabris E, Koopmans PC, Dambrink JE, Gosselink AT, Van't Hof AW, Ottervanger JP, Roolvink V, Remkes WS, van der Sluis A, Suryapranata H, Kedhi E. Fractional flow reserve-guided deferred versus complete revascularization in patients with diabetes mellitus. Am J Cardiol. 2016;118:1293-9.

29. Kennedy MW, Kaplan E, Hermanides RS, Fabris E, Hemradj V, Koopmans PC, Dambrink JH, Marcel Gosselink AT, Van't Hof AW, Ottervanger JP, Roolvink V, Remkes WS, van der Sluis A, Suryapranata H, Kedhi E. Clinical outcomes of deferred revascularisation using fractional flow reserve in patients with and without diabetes mellitus. Cardiovasc Diabetol. 2016;15:100.

30. Liu Z, Matsuzawa Y, Herrmann J, Li J, Lennon RJ, Crusan DJ, Kwon TG, Zhang M, Sun T, Yang S, Gulati R, Bell MR, Lerman LO, Lerman A. Relation between fractional flow reserve value of coronary lesions with deferred revascularization and cardiovascular outcomes in non-diabetic and diabetic patients. Int J Cardiol. 2016;219:56-62.

31. Kennedy MW, Fabris E, ljsselmuiden AJ, Nef H, Reith S, Escaned J, Alfonso F, van Royen N, Wojakowski W, Witkowski A, Indolfi C, Ottervanger JP, Suryapranata $\mathrm{H}$, Kedhi E. Combined optical coherence tomography morphologic and fractional flow reserve hemodynamic assessment of non-culprit lesions to better predict adverse event outcomes in diabetes mellitus patients: combine (oct-ffr) prospective study. Rationale and design. Cardiovasc Diabetol. 2016;15:144. 